\title{
Papers
}

\section{Multicentre randomised controlled trial of nasal diamorphine for analgesia in children and teenagers with clinical fractures}

\author{
Jason M Kendall, Barnaby C Reeves, Victoria S Latter on behalf of the Nasal Diamorphine Trial Group
}

\begin{abstract}
Objective To compare the effectiveness of nasal diamorphine spray with intramuscular morphine for analgesia in children and teenagers with acute pain due to a clinical fracture, and to describe the safety profile of the spray.

Design Multicentre randomised controlled trial. Setting Emergency departments in eight UK hospitals. Participants Patients aged between 3 and 16 years presenting with a clinical fracture of an upper or lower limb.

Main outcome measures Patients' reported pain using the Wong Baker face pain scale, ratings of reaction to treatment of the patients and acceptability of treatment by staff and parents, and adverse events. Results 404 eligible patients completed the trial (204 patients given nasal diamorphine spray and 200 given intramuscular morphine). Onset of pain relief was faster in the spray group than in the intramuscular group, with lower pain scores in the spray group at 5 , 10 , and 20 minutes after treatment but no difference between the groups after 30 minutes. $80 \%$ of patients given the spray showed no obvious discomfort compared with $9 \%$ given intramuscular morphine (difference $71 \%$, 95\% confidence interval $65 \%$ to $78 \%$ ). Treatment administration was judged acceptable by staff and parents, respectively, for $98 \%$ (199 of 203) and 97\% (186 of 192) of patients in the spray group compared with 32\% (64 of 199) and 72\% (142 of 197) in the intramuscular group. No serious adverse events occurred in the spray group, and the frequencies of all adverse events were similar in both groups (spray $24.1 \% v$ intramuscular morphine $18.5 \%$; difference $5.6 \%,-2.3 \%$ to $13.6 \%$ ).

Conclusion Nasal diamorphine spray should be the preferred method of pain relief in children and teenagers presenting to emergency departments in acute pain with clinical fractures. The diamorphine spray should be used in place of intramuscular morphine.
\end{abstract}

\section{Introduction}

Methods of giving analgesia to children are imperfect, particularly for those with moderate to severe acute pain. Oral analgesia is inadequate owing to limitations in drug choice and delayed gastric emptying. Intramuscular and intravenous injections can distress young people, and they are often restricted by nursing protocols. ${ }^{1}$ Rectal administration has limited acceptability and problems of slow and variable onset, and obtaining consent can be difficult. ${ }^{2}$

Giving drugs by the nasal route is well described and has several advantages. ${ }^{3}$ The nasal mucosa is richly vascularised, and the fenestrated epithelium drains by way of the facial and sphenopalatine veins, avoiding first pass metabolism. ${ }^{56}$ Diamorphine hydrochloride is highly soluble in water, facilitating its preparation at a high concentration. ${ }^{7}$ A small volume $(0.1 \mathrm{ml})$ can be used, promoting absorption transmucosally without major leakage down the back of the nose and subsequent swallowing. A study in a paediatric population showed better absorption of midazolam when given by nasal spray than when given by drops. ${ }^{8}$ Diamorphine given to children by the nasal route has only been described once. ${ }^{9}$ Other opioids have been given by this route (for example, fentanyl, meperidine ${ }^{10}{ }^{11}$ ) for postoperative pain.

Diamorphine hydrochloride has a potency about twice that of morphine salts and has a similar onset and duration of action. ${ }^{12}{ }^{13}$ Diamorphine powder that is snorted has a pharmacokinetic profile equivalent to that of diamorphine given intramuscularly. ${ }^{14}$ Therefore in the pilot study a dose of $0.1 \mathrm{mg} / \mathrm{kg}$ was used for diamorphine nasal drops compared with the standard treatment of $0.2 \mathrm{mg} / \mathrm{kg}$ for intramuscular morphine. ${ }^{9}$ Both treatments were observed to be effective and without side effects in 51 evaluable patients.

We aimed to compare the effectiveness of nasal diamorphine spray $(0.1 \mathrm{mg} / \mathrm{kg})$ with intramuscular morphine $(0.2 \mathrm{mg} / \mathrm{kg})$ for managing acute pain in children and teenagers with a clinical fracture, compare the reaction to treatment and acceptability of the two treatments, and evaluate the safety of the spray.

\section{Participants and methods}

Our study was a multicentre randomised controlled trial of a single dose of nasal diamorphine spray compared with intramuscular morphine for the management of acute pain in children and teenagers presenting to an emergency department with clinical fractures. The study was approved by the appropriate multicentre and local research ethics committees. Eight hospitals took

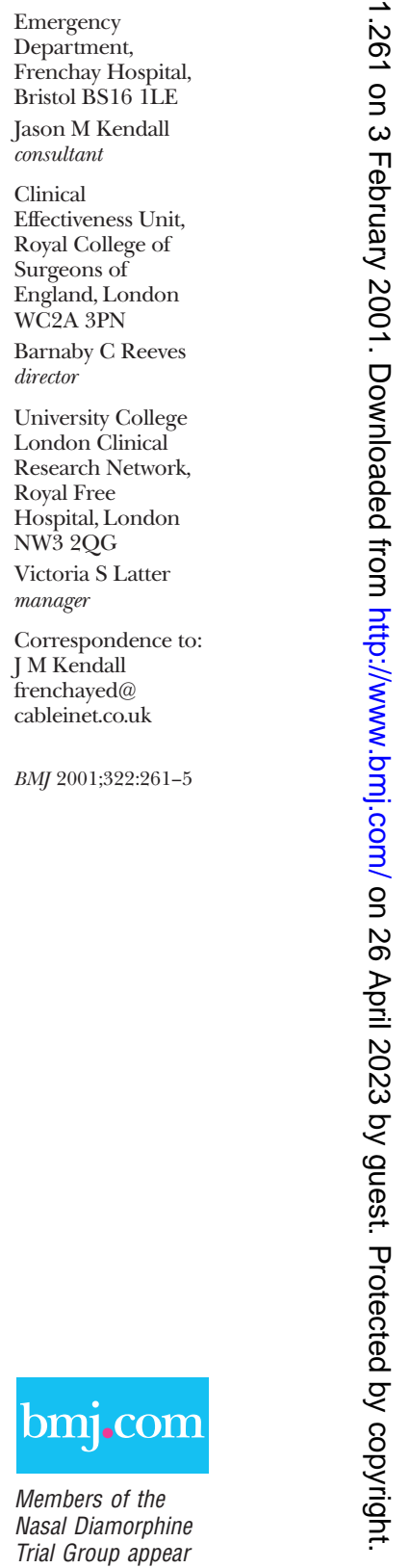
on the BMJ's website 
part-two teaching hospitals and six district general hospitals, with varying catchment populations.

\section{Population}

Patients aged between 3 and 16 years presenting to the emergency department with a clinical fracture of an upper or lower limb were eligible. The exclusion criteria were: not accompanied by a parent or guardian, head injury, need for immediate intravenous access, blocked nose or upper respiratory tract infection, learning difficulties, blindness or visual impairment, previous participation in the study, opioid analgesia in the preceding two days, and contraindications to diamorphine or morphine.

\section{Treatment allocation}

The patients were assessed promptly. Written informed consent was obtained from a parent or guardian. Patients were only considered for the trial if sufficient staff were available to allow recruitment to proceed quickly and there was no major delay in providing analgesia while consent was sought. Oral consent was also obtained from the patient if aged over seven years. When consent had been obtained and inclusion and exclusion criteria met, the next numbered case report form was opened. Randomised allocation codes, prepared before the start of the study by BCR, were concealed in sealed opaque envelopes in the case report form. Randomisation was blocked using blocks of unequal length and stratified by centre.

We were provided with freeze dried diamorphine hydrochloride in ampoules, diluent for reconstitution, and nasal dosing devices (to deliver $0.1 \mathrm{ml}$ of aerosolised drug). Diamorphine was reconstituted with diluent using a volume appropriate to the patient's weight to achieve a dose of $0.1 \mathrm{mg} / \mathrm{kg}$ in $0.1 \mathrm{ml}$. Morphine sulphate was given in a dose of $0.2 \mathrm{mg} / \mathrm{kg}$ intramuscularly in the conventional manner. If, in the opinion of the attending clinicians, the patient was still in extreme pain 20 or 30 minutes after treatment, rescue analgesia was offered as intramuscular morphine $(0.2 \mathrm{mg} / \mathrm{kg})$.

\section{Outcome measures}

Outcome measures assessed the effectiveness of pain relief, the patient's reaction to treatment administration, and the acceptability of the treatment to parents and staff.

Patients, parents, and staff assessed pain with the Wong Baker face pain scale (an ordinal scale of six faces ranging from smiling (score 1) to crying (score 6)) or a visual analogue scale, or both ${ }^{15}$; scores using the visual analogue scale were not completed by younger children because of difficulty in complying with this tool, and only the Wong Baker face pain scores are reported here. Pain was assessed at baseline and at 5 , 10, 20, and 30 minutes after treatment. All assessments were made without reference to previous assessments or those made by other observers.

At the time of giving treatment the nurse recorded the patient's reaction to administration according to one of five categories: no obvious discomfort, mild reaction, winced or withdrew, cried, and screamed. The nurse also described the acceptability of treatment as acceptable, stressful, very stressful, or unacceptable. A similar assessment of acceptability was made by the parent or guardian at 30 minutes. Pain scores were the primary outcome measure, and the patient's reaction to and acceptability of treatment were secondary outcomes.

Staff recorded any important observations or adverse events throughout the 30 minute period in accordance with the International Conference for Harmonisation's guidelines for good clinical practice. ${ }^{16}$ The intensity of adverse events were graded on a three point scale: mild, moderate, or severe. Pulse, respiratory rate, oxygen saturation, and the Glasgow coma score were also measured at each time point.

\section{Quality assurance}

Data from the case report forms were double entered into separate databases. At the close of the study the two databases were compared and discrepancies resolved by referring back to the case report forms. Any values that were out of range and that could not be checked from any other source were set to missing before data analyses.

\section{Statistical analysis}

Although pain score was the primary outcome, we also wanted to be able to comment on the risk of a serious adverse event. The target sample size of 200 in each group was chosen to exclude a serious rate for an adverse event in the spray group of greater than 18 in 1000 , if no such event was to be observed. This sample size gave ample power to detect a clinically important difference in pain score.

Analyses of outcomes between groups were carried out on an intention to treat basis, using two tailed $t$ tests for differences in continuous variables, $\chi^{2}$ tests for trend for ordinal variables (for example, Wong Baker face pain scores, Glasgow coma scores), and z tests for differences in proportions.

\section{Results}

\section{Study population}

Overall, 413 patients were recruited between July 1997 and September 1999. Three were excluded from

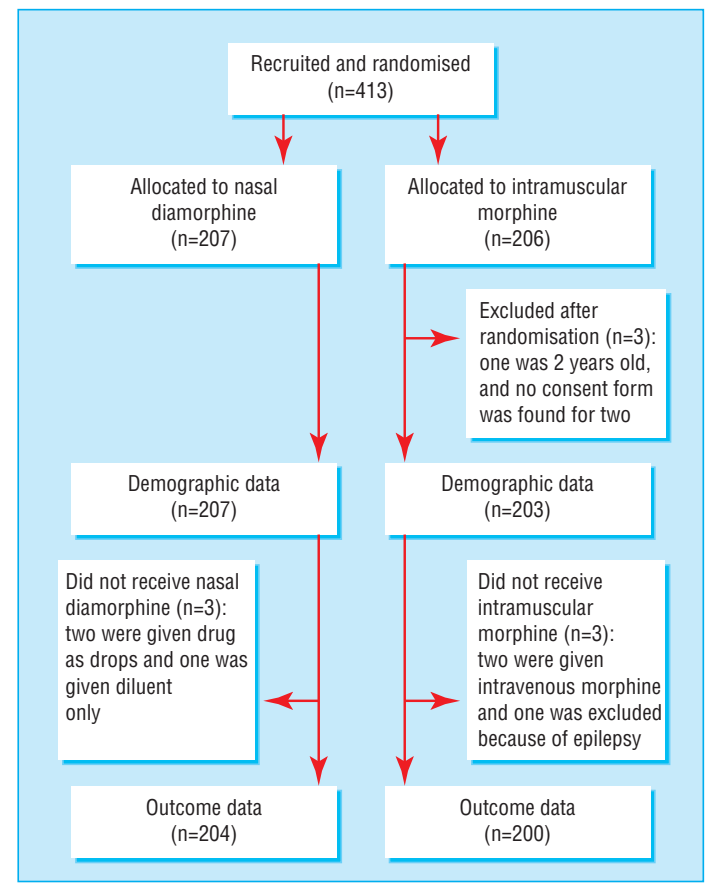

Fig 1 Flow of participants through trial 
Table 1 Characteristics of patients. Values are numbers (percentages) unless stated otherwise

\begin{tabular}{lcc} 
& $\begin{array}{c}\text { Nasal diamorphine } \\
\text { group (n=207) }\end{array}$ & $\begin{array}{c}\text { Intramuscular morphine } \\
\text { group ( } \mathbf{n}=\mathbf{2 0 3})\end{array}$ \\
\hline Mean (SD) age (months) & $115.3(41.0)$ & $108.0(38.7)$ \\
\hline Mean (SD) weight (kg) & $33.9(13.2)$ & $32.2(13.1)$ \\
\hline Male & $136(66)$ & $138(68)$ \\
\hline Injury sitet: & & \\
\hline Radius or ulna & $131(64)$ & $142(71)$ \\
\hline Supracondylar & $43(21)$ & $33(16)$ \\
\hline Tibia or fibula & $11(5)$ & $10(5)$ \\
\hline Other & $19(9)$ & $16(8)$ \\
\hline Hospital: & & \\
\hline A & $74(36)$ & $74(37)$ \\
\hline B & $23(11)$ & $25(12)$ \\
\hline C & $42(20)$ & $41(20)$ \\
\hline D & $13(6)$ & $13(6)$ \\
\hline E & $35(17)$ & $35(17)$ \\
\hline F & $11(5)$ & $7(3)$ \\
\hline G & $2(1)$ & $2(1)$ \\
\hline H & $7(3)$ & $6(3)$ \\
\hline Age & &
\end{tabular}

${ }^{*}$ Age not recorded for one patient given intramuscular morphine.

†Site of injury not recorded for three patients given spray and two given intramuscular morphine.

demographic analyses, and a further six were excluded from effectiveness and safety analyses because they did not receive either of the drugs in the study. Figure 1 shows the flow of patients through the trial. The characteristics of patients in both groups were well balanced (table 1).

\section{Effectiveness}

Both groups had similar distributions of Wong Baker face pain scores at the time of treatment $\left(\chi^{2}\right.$ test for trend $0.083, \mathrm{P}=0.77$; table 2 and fig 2). Pain scores improved over time in both groups, although the onset of analgesia was faster in the spray group. The distribution of pain scores for the spray group was lower than that for the intramuscular group at $5(4.29, \mathrm{P}=0.04)$, $10(8.74, \mathrm{P}=0.003)$, and 20 minutes $(9.84, \mathrm{P}=0.002)$ after treatment, but no different after 30 minutes (1.66, $\mathrm{P}=0.20)$. Pain scores assigned by parents and staff are not shown but were entirely consistent with the observations reported by patients (J M Kendall, personal communication).

The adequacy of analgesia, assessed by the need for rescue analgesia, did not differ between the groups: 9 and 10 children in the spray and intramuscular groups, respectively, required rescue analgesia at 20 minutes and 11 and 10 children at 30 minutes.

\section{Patient's reactions to treatment}

Patients reacted worse to intramuscular treatment than spray treatment $\left(\chi^{2}\right.$ test for trend 200.7; $\mathrm{P}<0.0001$; fig 3). Overall, $80 \%$ (162 of 203) of patients given the spray showed no obvious discomfort compared with $9 \%$ (17 of 199) given intramuscular morphine (difference $71 \%, 95 \%$ confidence interval $65 \%$ to $78 \%$ ). Conversely, 3\% (6 of 203) of patients screamed or cried when given the spray compared with $50 \%$ (99 of 199) when given morphine intramuscularly.

\section{Acceptability of treatment administration}

Acceptability, as measured by staff at the time of treatment, was significantly greater with the spray than with intramuscular morphine ( $\chi^{2}$ test for trend 167.4, $\mathrm{P}<0.0001)$. Treatment was judged acceptable by staff for 98\% (199 of 203) of patients in the spray group compared with $32 \%$ (64 of 199) in the intramuscular group (difference $66 \%, 59 \%$ to $72 \%$ ).

Acceptability, as measured by parents 30 minutes after treatment, was also significantly greater with the spray than with intramuscular morphine $\left(\chi^{2}\right.$ test for trend 43.1, $\mathrm{P}<0.0001)$. The method of pain relief was judged acceptable by parents for $97 \%$ (186 of 192) of patients in the spray group compared with $72 \%$ (142 of 197 ) in the intramuscular group (difference $25 \%, 32 \%$ to $78 \%$ ). The proportion of patients prepared to have the treatment again for future fractures was significantly higher for the spray $(94 \%)$ than for the intramuscular morphine (59\%; difference $35 \%, 28 \%$ to $43 \%$ ).

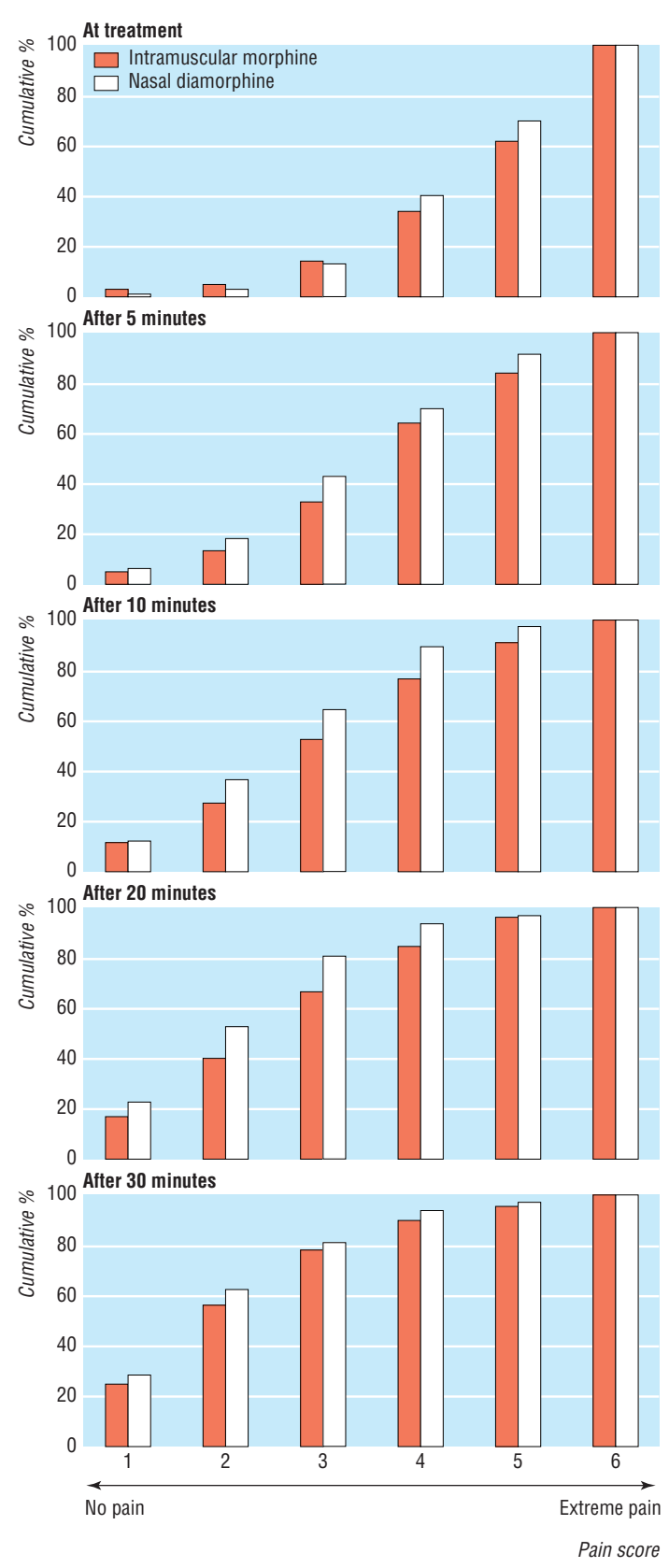

Fig 2 Cumulative percentages of patients' pain scores over time according to Wong Baker face pain scale (from 1 no pain to 6 severe pain) 
Table 2 Numbers (percentages) of children reporting Wong Baker face pain scores of differing severity (from 1 (no pain) to 6 (severe pain)) at baseline, 5, 10, 20, and 30 minutes after treatment. Denominators vary (190 to 197) as observations were missing for some patients at one or more time points

\begin{tabular}{|c|c|c|c|c|c|c|c|c|c|c|c|c|c|}
\hline \multirow{2}{*}{$\begin{array}{l}\text { Time } \\
\text { (minutes) }^{*}\end{array}$} & \multicolumn{6}{|c|}{ Nasal diamorphine group } & \multicolumn{6}{|c|}{ Intramuscular morphine group } & \multirow[b]{2}{*}{$\mathrm{P}$ value } \\
\hline & 1 & 2 & 3 & 4 & 5 & 6 & 1 & 2 & 3 & 4 & 5 & 6 & \\
\hline 0 & $2(1)$ & $4(2)$ & $19(10)$ & $51(26)$ & $55(28)$ & $66(34)$ & $5(2)$ & $5(2)$ & $18(9)$ & $39(20)$ & $55(28)$ & $74(38)$ & 0.77 \\
\hline 5 & $12(6)$ & $24(12)$ & $47(24)$ & $54(27)$ & $42(21)$ & $18(9)$ & $10(5)$ & $16(8)$ & $37(19)$ & $60(31)$ & $38(20)$ & $32(17)$ & 0.04 \\
\hline 10 & $23(12)$ & $49(25)$ & $54(28)$ & $49(25)$ & $16(8)$ & $5(3)$ & $22(11)$ & $31(16)$ & $50(26)$ & $46(24)$ & $29(15)$ & $17(9)$ & 0.003 \\
\hline 20 & $42(22)$ & $57(30)$ & $55(29)$ & $23(12)$ & $8(4)$ & $5(3)$ & $32(16)$ & $45(23)$ & $52(27)$ & $35(18)$ & $23(12)$ & 7 (4) & 0.002 \\
\hline 30 & 54 (28) & 65 (34) & 36 (19) & 24 (13) & 7 (4) & $5(3)$ & $48(25)$ & 61 (32) & $42(22)$ & $23(12)$ & $11(6)$ & $8(4)$ & 0.20 \\
\hline
\end{tabular}

* Mean time at which observations were made did not differ significantly between groups (intramuscular morphine - nasal diamorphine mean observation times: 5 minutes, difference $=0.2$ minutes, $t=0.86, P=0.39 ; 10$ minutes, difference $=0.6$ minutes, $t=1.24, P=0.21 ; 20$ minutes, difference $=0.3$ minutes, $t=0.58, P=0.56 ; 30$ minutes, difference $=0.4$ minutes, $t=0.51, P=0.61$.

$+\chi^{2}$ tests for trend.

Safety

No difference was found for pulse, respiratory rate, and Glasgow coma score between the groups at any time. Although not clinically important, median oxygen saturation was slightly lower in the spray group at 5,10 , and 20 minutes, with no difference at baseline or 30 minutes. Only $8 \%$ (17 of 201) of patients in the spray group and $11 \%$ (22 of 200) in the intramuscular group had an oxygen saturation less than $95 \%$ at any time between treatment and the 30 minute observation period.

No unexpected adverse events were observed. One patient in the intramuscular group had nausea and vomiting (a serious adverse event of moderate intensity), was admitted for a brief period of observation, and recovered spontaneously. Overall, 84 non-serious adverse events occurred; all were mild except for one in the spray group that was considered severe (abdominal pain and vomiting). Overall, 24\% (49 of 203) of patients in the spray group had an adverse event compared with 19\% (37 of 200) in the intramuscular group (difference 5.6\%,-2.3\% to $13.6 \%$ ). Over half of the adverse events involved irritation at the site where the drug was given.

\section{Discussion}

Nasal diamorphine spray $(0.1 \mathrm{mg} / \mathrm{kg})$ provides the same degree of pain relief as intramuscular morphine $(0.2 \mathrm{mg} / \mathrm{kg})$, and the spray provides quicker onset of pain relief than intramuscular morphine. Young

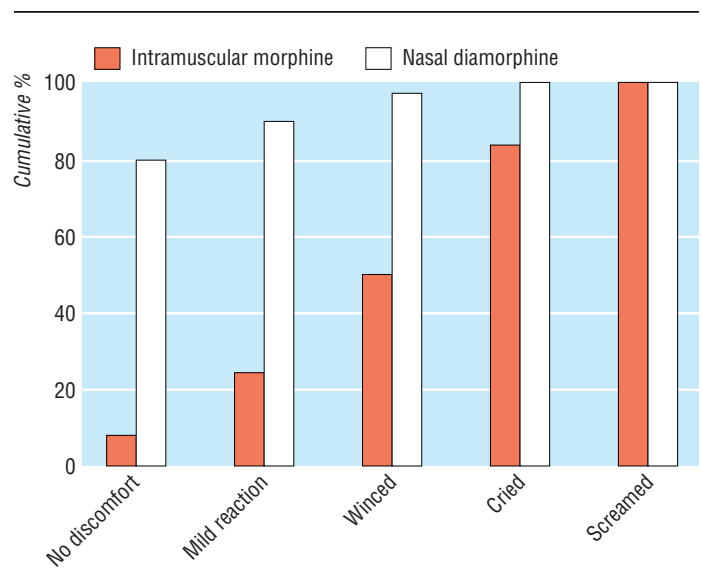

Fig 3 Cumulative percentages of patients' reactions to treatment administration people tolerated treatment better by spray than by the intramuscular route. The spray was judged more acceptable than intramuscular morphine by both staff and parents. Nasal diamorphine spray had an acceptable safety profile in 204 patients in our study.

We believe that these findings are valid and widely applicable. The significance levels for tests of difference in outcomes between groups indicate that the findings of effectiveness are extremely unlikely to arise by chance. The balance achieved between groups in the characteristics of patients and stratification by hospital suggests that randomisation was well concealed, ruling out significant confounding.

The finding of quicker onset of pain relief was somewhat obscured by the wide distribution of pain scores at each time point. This finding is, however, supported by the faster onset of oxygen desaturation in the spray group-that is, the time course of the differences between groups in this objective physiological measurement closely matches that for pain relief.

A degree of bias is possible because patients, parents, and staff could not be blinded to the method of pain relief. It was considered unethical to adopt a "double dummy" study design; such a design would also have precluded measurement of differences between groups in patients' reactions to treatment and acceptability of that treatment to parents and staff. We acknowledge that the strength of the difference between the two groups in the patients' reactions to treatment and acceptability, as judged by the parents and staff, may well arise in part from an intrinsic antipathy towards giving young people injections.

Bias was unlikely to explain the differences in pain scores reported by the patients themselves because the faster onset of pain relief reported by the patients in the spray group was mirrored by the trend in oxygen saturation, an objective physiological measure. A tendency for patients in the spray group to rate their pain as less severe because of the greater acceptability to them of this type of treatment might account for some of the difference in reported pain between the groups. If true, we argue that this should be considered part of the effect of the intervention rather than the result of bias, because any such effect persists outside the context of the study.

We were unable to record the total number of eligible patients presenting to participating emergency departments during the recruitment period because of the busy nature of the setting. It is almost certain that only a minority of eligible patients were recruited because there were no dedicated research staff in cen- 
What is already known on this topic

All current methods for giving analgesia to young people in acute pain have limitations

\section{What this study adds}

Diamorphine given by the nasal route resulted in more rapid analgesia than intramuscular morphine in young people in acute pain

Patients tolerated the spray better than the intramuscular injection, and parents and staff found the spray more acceptable

The safety profile of the spray was acceptable, with no serious adverse events reported

Nasal diamorphine should be preferred to intramuscular morphine

tres, and recruitment depended on the motivation of local staff. Nevertheless, we believe that the results are widely applicable because the reasons for not recruiting patients-for example, department too busy, shortage of staff, motivation of staff on duty-are unlikely to be related to the characteristics of the patients. That the study was carried out in several hospitals (two teaching hospitals and six district general hospitals, including one paediatric emergency department, with varying catchment populations) also supports the applicability of the findings.

The most common side effects of opioids are nausea, vomiting, constipation, and drowsiness. Respiratory depression is sometimes seen at higher doses. The side effect profiles of nasal diamorphine spray and intramuscular morphine in our study did not differ from each other either qualitatively or quantitatively, except for oxygen desaturation. Although the onset of oxygen desaturation was statistically quicker with the spray than with intramuscular morphine, the difference was not clinically important. We cannot rule out the possibility that the spray may, rarely, cause a serious adverse event; as in many randomised controlled trials, a study designed to detect a difference in serious adverse events would have required a sample size that would not have been feasible to recruit. Because no serious adverse events were observed in the spray group, however, we can confidently conclude that the rate of serious adverse events was less than 18 in 1000 .

Nasal diamorphine spray may be the best way to provide analgesia for young people in different circumstances-for example, those with painful burns or finger tip injuries and those who require dressing changes. Indeed, outside of this trial, nasal diamorphine spray has been and is being used for these purposes in many of the study centres. The spray is also currently being evaluated in adults for the control of breakthrough pain in patients receiving palliative care and in surgical patients for postoperative analgesia.

\section{Conclusion}

Nasal diamorphine spray is a safe and effective method of pain relief for young people presenting to emergency departments in acute pain with clinical fractures, and it should be preferred to intramuscular morphine. There should no longer be any reason to give intramuscular morphine to such children because the spray is appropriate wherever intramuscular morphine is being considered.

We thank the patients, parents, and staff; Dr P Younge, Mr M Nicol, Dr P Davies, Dr D Williamson, Mr J Benger, Mr S Cope, Miss C Taylor, Dr D Boon, Dr S Odum, and Dr J Louis who were involved in data collection; Clare Swinburn who created the study database; and Sandra Osmond (Research and Development Support Unit, Bristol Royal Infirmary), and Joanne Shill, Tracy Walker, and Julie Ellis (CP Pharmaceuticals) who entered the data. CP Pharmaceuticals provided the drugs for the study.

Contributors: JMK participated in the design, recruitment, and clinical aspects of the study. BCR participated in the design and analysis of the study. VSL participated in the design and quality assurance. All authors contributed to the writing of the paper and will act as guarantors.

Funding: CP Pharmaceuticals contributed to the costs of data management and analysis.

Competing interests: BCR received a consultancy fee from CP Pharmaceuticals for cleaning and analysis of data, which was paid into a research fund at the Royal College of Surgeons. CP Pharmaceuticals did not contribute to the paper. VSL worked for CP Pharmaceuticals during the study design and collection phases of the data but left before the paper was written.

1 Simini B. Patients' perceptions of pain with spinal, intramuscular, and venous injections. Lancet 2000;355:1076

2 Mitchell J, James MA, Lunn JN. A fundamental problem of consent. BMJ 1995;310:43-6.

3 Karl HW, Keiffer AT, Rosenberger JL, Larach MG, Ruffle JM. Comparison of the safety and efficacy of intranasal midazolam and sufentanil for preinduction of anaesthesia in paediatric patients. Anaesthesiology 1992;76:209-15.

4 Walbergh EJ, Wills RJ, Eckliert J. Plasma concentrations of midazolam in children following intranasal administration. Anaesthesiology 1991;74:233.

5 Cauna N, Hinderer KH. Fine structure of blood vessels of human respiratory mucosa. Ann Otol Rhinol Laryngol 1969;78:865.

6 Williams PL, Warwick R. Gray's anatomy, 36th ed. London: Churchill Livingstone, 1980 .

7 Reynolds JEF. Martindale: the extra pharmacopoeia, 32nd ed. London: Royal Pharmaceutical Society, 1999.

8 Ljung B, Andréasson S. Comparison of midazolam nasal spray to nasal drops for the sedation of children. J Nucl Med Technol 1996;24:32-4.

9 Wilson JA, Kendall JM, Cornelius P. Intranasal diamorphine for paediatric analgesia: assessment of safety and efficacy. J Accid Emerg Med 1997; 14:70-2.

10 Striebel HW, Pommerening J, Riefer A. Intranasal fentanyl titration for postoperative pain management in an unselected population. Anaesthesia 1993;48:753-7.

11 Striebel HW, Malewicz J, Hermanns K, Castell R. Intranasal meperidine titration for postoperative pain relief. Anesth Analg 1993;76:1047-51

12 Robinson SL, Rowbotham DJ, Smith G. Morphine compared with diamorphine. A comparison of dose requirements and side effects after hip surgery. Anaesthesia 1991:46:538-40.

13 British Medical Association and Royal Pharmaceutical Society of Great Britain. British national formulary 39, Mar 2000.

14 Cone EJ, Holicy BA, Grant TM, Darwin WD, Goldberger BA Pharmacokinetics and pharmacodynamics of intranasal "snorted" heroin.J Analyt Toxicol 1993;17:327-37.

15 Huskisson EC. Measurement of pain. Lancet 1974;ii:1127-31.

16 ICH Secretariat. ICH harmonised tripartite guideline for good clinical practice. Surrey: Brookwood Medical, 1996.

(Accepted 20 October 2000)

\section{Correction}

The Ghost of Christmas Past: health effects of poverty in London in 1896 and 1991

The penultimate paragraph of the Results section in this paper by Dorling et al (23-30 December, pp 1547-51) should read: "The results of further correlation analyses suggest that for deaths under the age of 65 the modern index makes a slightly greater contribution to predicting all cause mortality in 1991-5 $(r=0.56, \mathrm{P}<0.001)$ than does the Booth index $(r=0.46, \mathrm{P}<0.001)$. This is substantiated by the results of the partial correlation analyses, where $r=0.39$ $(\mathrm{P}<0.001)$ when mortality is correlated with the modern [not the Booth] index, controlling for the Booth [not the modern] index, but $r=0.08(\mathrm{P}=0.36)$ when mortality is correlated with the Booth index, controlling for the modern index." 